\title{
Ambulation monitoring of transtibial amputation subjects with patient activity monitor versus pedometer
}

\author{
Nancy L. Dudek, MD, MEd; Omar D. Khan, MD; Edward D. Lemaire, PhD; Meridith B. Marks, MD, MEd; \\ Leyana Saville \\ Division of Physical Medicine and Rehabilitation, The Rehabilitation Centre, University of Ottawa, Ottawa, Canada
}

\begin{abstract}
Our study aimed to compare the accuracy of step count and ambulation distance determined with the Yamax Digi-Walker SW-700 pedometer (DW) and the Ossur patient activity monitor (PAM) in 20 transtibial amputation subjects who were functioning at the K3 Medicare Functional Classification Level. Subjects completed four simulated household tasks in an apartment setup and a gymnasium walking course designed to simulate outdoor walking without the presence of environmental barriers or varied terrain. The mean step count accuracy of the DW and the PAM was equivalent for both the household activity (75.3\% vs $70.6 \%)$ and the walking course (93.8\% vs $94.0 \%)$. The mean distance measurement accuracy was better with the DW than with the PAM (household activity: $72.8 \%$ vs $0 \%$, walking course: $92.5 \%$ vs $86.3 \%$; $p<0.05$ ). With acceptable step count accuracy, both devices are appropriate for assessing relatively continuous ambulation. The DW may be preferred for its more accurate distance measurements. Neither device is ideal for monitoring in-home ambulation.
\end{abstract}

Key words: activities of daily living, ambulation, ambulatory monitoring, amputees, gait, measurement, pedometer, rehabilitation, step count, validation studies.

\section{INTRODUCTION}

Walking is one of the most fundamental components of daily physical activity. The importance of monitoring daily ambulation and activities in people who have undergone lower-limb amputation has been identified previously [1-4]. Accurate monitoring of ambulation levels has the potential to facilitate better care of amputation patients by assisting clinicians and researchers prescribe and choose therapies, measure the effects of interventions, choose the most appropriate prosthetic components, and predict postamputation abilities.

The effects of rehabilitation interventions such as changing a prosthetic component or adding a gait aid may be more objectively measured with a reliable and accurate ambulation monitoring device. Prescription of an appropriate prosthesis would also be facilitated if detailed and objective information regarding daily activity were available. For example, the extent of prosthetic use can influence the choice of prosthetic foot for individual patients. Determining objective time lines for replacing prosthetic devices would also be facilitated, similar to the use of an automotive odometer to direct regular vehicle maintenance. Finally, activity monitoring would facilitate prosthetic research if a valid and reliable measure of functional activity were available.

Activity level categorization in those who have undergone lower-limb amputation is commonly performed using the Medicare Functional Classification Level (MFCL) [5].

Abbreviations: $\mathrm{BMI}=$ body mass index, DW $=$ Yamax DigiWalker SW-700 pedometer, MFCL = Medicare Functional Classification Level, PAM = Össur patient activity monitor, SAM = step activity monitor.

*Address all correspondence to Nancy L. Dudek, MD, MEd; 505 Smyth Road, Room 1105D, Ottawa, ON K1H 8M2, Canada; 613-737-7350, ext 75596; fax: 613-737-9638. Email: ndudek@ottawahospital.on.ca

DOI: 10.1682/JRRD.2007.05.0069 
The MFCL is used by the physician and prosthetist to determine the patient's ability to reach a "defined functional state within a reasonable period of time" [5] and to choose the most appropriate prosthetic device for his or her functional level. The MFCL categorizes lower-limb amputees into broad levels, such as nonambulator (K-level 0), household ambulator (K-level 1), limited community ambulator (K-level 2), community ambulator (K-level 3), and high-level user (K-level 4). Within each functional level, considerable variability in daily activity levels can exist between individual patients; however, the MFCL cannot subcategorize individuals within a functional level. Also, categorization is based on the person's ambulation potential, often obtained through patient self-report of abilities, not his or her actual daily activities.

Patient self-report has been used in several questionnaires to measure prosthetic use in those with lower-limb amputation. Questionnaires such as the 36-Item Short Form [6], the Amputee Activity Survey [7], and the Locomotor Capabilities Index [8] have established validation properties in this population. However, self-report has been criticized as being inaccurate owing to considerable subjectivity [9]. The limiting components of most selfreport assessments are their imprecision in determining small but important differences in activity level and their inability to quantify amputee activity. Currently, selfreport scales are not considered accurate or sensitive enough for use in research requiring highly accurate monitoring of amputation patient activity [10]. Inclusion of an objective and accurate activity-monitoring device in studies attempting to validate self-report scales would prove invaluable in determining the responsiveness and applicability of these scales.

The importance of identifying an objective and accurate activity monitor for those with lower-limb amputation has been previously recognized [1-3]. Initial efforts were made by Holden et al. with their work on a step monitor consisting of a foot switch, storage unit, and retrieval unit [1]. Although this device was shown to measure activity accurately, it was impractical for largerscale patient use owing to limited data acquisition capabilities and the need to modify the prosthesis to incorporate the device. Activity has subsequently been studied using a variety of pedometers and accelerometers, each with its own advantages and disadvantages. An ideal device has not yet been found.

Pedometers are devices that are typically worn at the waist and are capable of counting steps. They have the advantage of being relatively inexpensive and readily available. The importance of step count as a relevant measure of physical activity level in nondisabled people has been shown [11]. Pedometer accuracy in this population has been assessed, and the Yamax SW series have been shown to be among the most accurate and affordable models commercially available [12-13]. High measurement variability for those with gait disorders has been considered a disadvantage of pedometer-based ambulation monitoring in the disabled population [14]. However, in people with relatively normal gait patterns, such as those with transtibial amputation who ambulate at the active community level (K3 and K4), the use of pedometers should remain a potential option.

Accelerometers can be used to establish patterns of movement during activity by comparing rates of acceleration from sensors placed at different areas of the body. Some accelerometer models have been validated in the amputee population [3,9,15]. Their bulky design [9], need for customization, and relatively high cost [2] make their use less practical for community-based studies.

The Össur patient activity monitor (PAM) (Reykjavík, Iceland) attaches to a prosthesis and uses inertial sensors to measure angle and angular velocity of the lower leg. Through digital signal processing, it uses these data to calculate stride and activity parameters. Bussmann et al. determined that the PAM was a valid device for classifying everyday activities of people with transtibial amputation into inactive, active, and locomotion categories [16]. While the authors presented data on stride count for treadmill walking and overground walking, they did not assess the accuracy of step count and ambulation distance during typical household daily activities. If the PAM is to be worn by people with amputation for community activity monitoring, its accuracy during continuous and noncontinuous ambulation in indoor and outdoor environments must be determined.

The objective of our study was to measure and compare the accuracy of step count and ambulation distance determined with the Yamax Digi-Walker SW-700 pedometer (DW) (New-Lifestyles, Inc; Lee's Summit, Missouri) and the PAM in transtibial amputation subjects at the K3 level within a simulated apartment setting and during relatively continuous gymnasium walking. 


\section{METHODS}

\section{Instruments}

The PAM measures and displays distinct data parameters related to activity level, including inactivity time, nonlocomotor activity time, locomotion time, distance traveled, steps taken, and speeds traveled. The device provides three output activity classes-inactive, active, and locomotion-by applying a stride-detection algorithm to the steps taken. If the subject is stationary for more than $20 \mathrm{~s}$, he or she is classified as inactive. If the subject takes more than 10 strides in a row, the subject is classified as locomotion, starting from the first stride taken. When the subject is not in either of these two classes, he or she is classified as active. The active classification therefore includes a mix of stepping and not stepping associated with everyday movement tasks performed in a limited space, such as within a home environment.

The DW was chosen for this study as the metric version of the Yamax SW series. The DW measures step count and when preset with the subject's step length (in meters) and weight (in kilograms) can estimate ambulation distance and calorie expenditure based on the step count data.

\section{Participants}

We reviewed physician clinic notes for all patients who had undergone transtibial amputation and who were seen in the outpatient clinic at The Rehabilitation Centre, Ottawa, Canada, between January 1, 1998, and March 31, 2005 (249 patients). The charts of those with unilateral amputation who were identified as community-level ambulators were extracted (175 patients). Three of the study investigators (Drs. Dudek, Kahn, and Marks) independently reviewed these charts and identified those ambulating at the K3 level. Consensus among all three investigators was used to identify all $\mathrm{K} 3$ ambulators as potential study participants (69 patients).

An introductory letter was mailed to each potential participant, and study recruitment was conducted through a follow-up telephone call within 1 to 2 weeks. The order in which participants were contacted was determined by random number generation. Patients who agreed to take part in the study were screened with questions about their current prosthetic use and medical stability to ensure that no recent changes in their functional status had occurred. Exclusion criteria included prosthesis malfunction or new medical comorbidities affecting ambulation.
All subjects provided informed consent to take part in the study. The experimental protocol was approved by the Research Ethics Board at The Rehabilitation Centre.

\section{Procedures}

\section{Subjects}

Testing took place at The Rehabilitation Centre during one visit in July or August 2005. Basic demographic data were collected for each subject. Each subject's prosthetic device was reviewed by the principal investigator using a list of screening questions developed by two certified prosthetists. Once prosthetic safety issues were ruled out, subjects could continue with the study protocol.

Subjects were taken to a fully equipped one-bedroom apartment setup within The Rehabilitation Centre to perform simulated household activities. The floor covering was low shag carpet. We determined step length using a distance wheel that measured 10 steps walked at a selfdetermined speed. The calculated step length for each subject was entered into the DW before testing.

\section{Device Application}

The PAM was placed anteriorly on the prosthesis, in the sagittal plane, in line with the second digit, and at a height of $20 \mathrm{~cm}$ from the bottom of the device to the floor. The device was affixed to the prosthesis with its self-adhesive strap and a $2.5 \times 5.0 \mathrm{~cm}$ piece of doublesided adhesive tape to avoid any bouncing or sliding of the unit during testing. The DW was attached over the subject's belt or waistband using the clip at the back of the device. It was positioned vertically midway between the subject's lateral hip and umbilicus to estimate the position of the anterior superior iliac spine. Both devices were placed on the same side as the prosthesis. If abdominal obesity resulted in a less than optimal position (i.e., device not positioned vertically), the DW was moved approximately $7 \mathrm{~cm}$ laterally at or slightly above waist level.

\section{Simulated Household Activities}

Subjects observed the principal investigator during a demonstration and then completed four simulated household tasks (Figure):

1. Subjects started seated at the right side of the bed, stood up, and walked to the bathroom sink. At the sink, subjects simulated brushing their teeth for $20 \mathrm{~s}$ and 


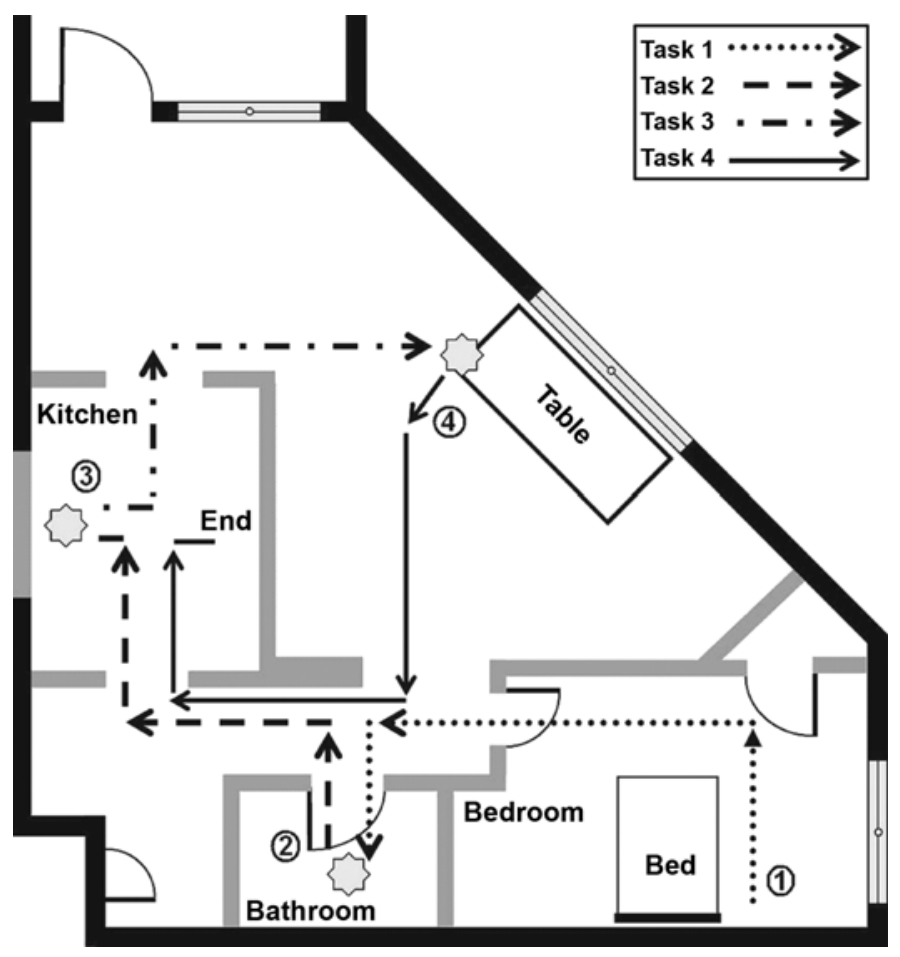

Figure.

Schematic of layout of apartment and four tasks performed.

then brushing their hair for $20 \mathrm{~s}$. Subjects washed their hands for $15 \mathrm{~s}$ and dried them at the towel rack.

2. From the bathroom, subjects walked into the kitchen, removed three dishes from the cabinet, and placed them on the counter below. Subjects then filled the kettle with water, placed it back on the stove, removed two slices of bread from a loaf of bread, and placed them in the toaster.

3. Subjects walked from the kitchen into the dining room and sat at the table, where a simulated meal setup was present. Subjects buttered two slices of toast, sprinkled salt and pepper over a simulated meal, and poured a glass of water from a pitcher. Each task item was placed across the table from the subjects, requiring them to reach the trunk and arms forward to pick up the items.

4. Subjects rose from the chair in the dining room, walked back into the kitchen, and washed a set of dishes and utensils in the sink.

We videotaped subjects to obtain criterion step count data. The distance traveled was measured by a research assistant following the subject with a measurement wheel. We calculated walking speed using an average of the walking speeds during tasks 1 and 4, where longer walking distances occurred. We calculated walking time using the video data and walking distance using the measurement wheel.

\section{Distance Walking Task}

The walking and turning task was designed to simulate outdoor walking without the presence of environmental barriers (i.e., curbs, stairs, and ramps) or varied terrain. Testing was completed in The Rehabilitation Centre's gymnasium, which has a tiled floor.

Each subject was instructed to walk 10 lengths of the gymnasium, marked out at $16 \mathrm{~m}$ from end to end, for a total of $160 \mathrm{~m}$. Subjects were instructed to walk at the speed they normally would during a typical day. At each end of the gymnasium, subjects were instructed to turn safely and directly on or as close to the marker points as possible. A precise method of turning was not specified to the subjects. We videotaped and timed subjects during the gymnasium walking to obtain criterion data for comparison to the data collected from the PAM and DW.

\section{Data Analysis} by

The percent accuracy of the devices was calculated

$$
100 \angle\left(\frac{\text { Device } \angle \text { Criterion }}{\text { Criterion }} \times 100\right)
$$

The accuracy of the step count in the apartment was based only on steps taken during ambulation (which we defined as two or more steps taken consecutively for the purpose of spatial relocation). We used descriptive statistics to calculate the mean and standard deviation of the various parameters. We used $t$-tests for equality of mean values for interdevice comparisons. We analyzed the data using SPSS, version 11.5 for Windows (SPSS, Inc; Chicago, Illinois).

\section{RESULTS}

A total of 23 patients agreed to take part in the study. Three patients subsequently chose not to participate for personal reasons unrelated to the exclusion criteria. Thus, 20 subjects were tested using the study protocol.

Participant characteristics are summarized in Table 1. Obesity, defined as body mass index (BMI) $\geq 30$, was the most common comorbidity, followed by diabetes mellitus. 
Table 1.

Participant characteristics $(n=20)$. Data presented as mean \pm standard deviation (range) or total number and percentage of total where appropriate.

\begin{tabular}{|c|c|}
\hline Demographic & Value \\
\hline Age (yr) & $58.6 \pm 10.8(43-82)$ \\
\hline \multicolumn{2}{|l|}{ Sex } \\
\hline Male & $13(65)$ \\
\hline Female & 7 (35) \\
\hline Height (cm) & $171.2 \pm 8.9(153-185)$ \\
\hline Weight (kg) & $82.8 \pm 16.3(57.6-122.5)$ \\
\hline Body Mass Index & $28.3 \pm 5.3(22-40)$ \\
\hline \multicolumn{2}{|l|}{ Education Level } \\
\hline Secondary & $4(20)$ \\
\hline Postsecondary & $16(80)$ \\
\hline \multicolumn{2}{|l|}{ Side of Amputation } \\
\hline Left & $12(60)$ \\
\hline Right & $8(40)$ \\
\hline Time Since Amputation (yr) & $26.7 \pm 20.0(2-62)$ \\
\hline \multicolumn{2}{|l|}{ Etiology of Amputation } \\
\hline Trauma & $12(60)$ \\
\hline Vascular & $4(20)$ \\
\hline Diabetes Mellitus & $1(5)$ \\
\hline Tumor & $1(5)$ \\
\hline Congenital & $1(5)$ \\
\hline Other & $1(5)$ \\
\hline \multicolumn{2}{|l|}{ Comorbidity } \\
\hline Obesity & $7(35)$ \\
\hline Diabetes Mellitus & $5(25)$ \\
\hline Peripheral Vascular Disease & $3(15)$ \\
\hline Renal Failure & $1(5)$ \\
\hline Smoker & $5(25)$ \\
\hline Gait Aid Used Outdoors & $6(30)$ \\
\hline Step Length (m) ${ }^{*}$ & $0.65 \pm 0.07(0.58-0.83)$ \\
\hline \multicolumn{2}{|l|}{ Speed $(\mathrm{m} / \mathrm{s})^{*}$} \\
\hline Apartment & $0.93 \pm 0.11(0.79-1.20)$ \\
\hline Gymnasium & $1.13 \pm 0.14(0.88-1.52)$ \\
\hline
\end{tabular}

A minority of the subjects (30\%) required a single-point cane for outdoor ambulation. Two subjects chose to use a single-point cane during ambulation in the gymnasium. None of the subjects used a gait aid in the apartment.

\section{Step Count}

Analysis of the videotaped data from the apartment setting revealed steps of varying length and style. In addition to steps taken in a symmetrical fashion for the purpose of ambulation from one location to another, sub- jects routinely sidestepped and stepped forward and then back using the same foot and occasionally used shuffling steps while performing components of the simulated daily activities.

The mean step count percent accuracy for the DW was $75.3 \pm 20.9$ for the apartment and $93.8 \pm 6.7$ for the gymnasium (all data presented as mean \pm standard deviation unless otherwise noted). For the PAM, the accuracies were $70.6 \pm 10.1$ for the apartment and $94.0 \pm 4.0$ for the gymnasium. No statistically significant difference was noted in step count accuracy between the devices in the apartment or the gymnasium ( $p>0.05)$. However, both the DW and PAM were statistically different when compared with the criterion values for step count $(p<0.05)$ (Table 2).

\section{Ambulation Distance}

Table 2 illustrates the criterion values for distance in the gymnasium and apartment as compared with the values recorded for distance by the DW and PAM. The PAM failed to display any distance measurements during apartment testing. The criterion values were significantly different for the PAM $(p<0.05)$ but not for the DW ( $p>$ 0.05 ). The DW's percent accuracy for distance was $72.8 \pm$ 18.2 for the apartment and $92.5 \pm 6.3$ for the gymnasium. The PAM's accuracy for distance in the gymnasium was $86.3 \pm 7.7$. The DW was significantly more accurate than the PAM for ambulation distance in both the apartment and the gymnasium $(p<0.05)$.

\section{Effect of Body Habitus}

Obesity affected the accuracy of the DW but not the PAM. Significant differences in step count accuracy with

Table 2.

Comparison of criterion and device values. Data presented as mean \pm standard deviation.

\begin{tabular}{lccc}
\hline $\begin{array}{c}\text { Test } \\
\text { Environment }\end{array}$ & $\begin{array}{c}\text { Criterion } \\
\text { Value }\end{array}$ & DW & PAM \\
\hline Apartment & & & \\
Step Count & $82.3 \pm 8.1$ & $64.5 \pm 20.9$ & $58.0 \pm 9.5$ \\
Distance (m) & $43.2 \pm 2.1$ & $40.5 \pm 14.3^{*}$ & 0 \\
Gymnasium & & & \\
Step Count & $248.1 \pm 23.6$ & $233.0 \pm 29.0$ & $236.3 \pm 28.7$ \\
Distance (m) & 160.0 & $156.0 \pm 15.3^{*}$ & $143.0 \pm 18.9$ \\
*Not statistically different from criterion value $(p>0.05)$. \\
$\begin{array}{l}\text { DW = Yamax Digi-Walker SW-700 pedometer, PAM = Össur patient activity } \\
\text { monitor. }\end{array}$ \\
\hline \hline
\end{tabular}


the DW were found between obese and nonobese subjects in both the apartment $(63.5 \% \pm 18.1 \%$ vs $83.2 \% \pm$ $19.4 \%, p<0.05)$ and the gymnasium $(90.2 \% \pm 8.1 \%$ vs $96.2 \% \pm 4.6 \%, p<0.05)$.

\section{DISCUSSION}

The objectives of this study were to determine the validity of the DW and PAM for subjects with unilateral transtibial amputation who were ambulating at the $\mathrm{K} 3$ level in a simulated apartment setting and during relatively continuous walking and to compare the accuracy of the two devices. If a device is to be useful for monitoring daily ambulation, it needs to be accurate in an indoor environment, since most daily activities occur in this setting. Our study protocol incorporated a typical household environment and a simulated simple walking environment (i.e., no environmental barriers or uneven terrain). These test environments are likely representative of ambulation within a small store or office space (apartment) and ambulating down the hallways of a hospital, large office building, or shopping center (gymnasium). To our knowledge, validation of the PAM and a pedometer during daily household tasks using videotaped reference data had not previously been documented in the literature.

In general, both devices were less accurate in the apartment than in the gymnasium setting. Similar underestimations in step count were observed with both. Although distance was underestimated by both devices, the DW was more accurate than the PAM, which failed to register any distance during testing in the apartment. These findings were likely due to factors related to the types of activities performed during the apartment protocol. In the apartment, subjects performed a variety of daily activities over relatively short distances. Therefore, the length and speed of steps varied considerably depending on the type of activity being performed. Many subjects also incorporated sidestepping while performing tasks at the kitchen counter. The devices, most significantly the PAM, appear to be less adept at measuring distances in situations involving varied types of steps.

The reasons for underestimation of distance in the presence of varied steps are not completely clear. With the PAM, the discrepancies may to be related to internal settings for activity classification and the types of activities encountered in the apartment. When the PAM encounters 10 consecutive steps, it begins classifying the activity as locomotion. If the subject continues to step consecutively, the PAM will continue to register the activity as locomotion. Ambulation distance is calculated by the sum of all the stride lengths measured by the unit's inertial sensors. Review of the apartment videotape data revealed that subjects took on average 12 to 16 steps to ambulate from one task to the next in the apartment. These relatively short distances, interrupted by missed steps, may have led the device to misclassify the activity as "active" instead of "locomotion" and, in so doing, introduced measurement errors. Bussmann et al. also noted that missing one stride caused misclassification of the activity being performed [16]. We agree with their suggestion that lowering the setting of 10 consecutive steps may further improve the classification accuracy of the device.

Another possible explanation is subject walking speed. The PAM was designed to function optimally at walking speeds above $0.75 \mathrm{~m} / \mathrm{s}$; below this speed, accuracy decreases significantly [16]. We chose subjects at the K3 level for this study to ensure that participants were likely to ambulate above $0.75 \mathrm{~m} / \mathrm{s}$. The speed at which subjects ambulated in the gymnasium and apartment setting was calculated to be above this critical level for all subjects. Therefore, ambulation speed did not appear to explain the inaccuracy of the PAM in the apartment settings. To further verify this result, we performed several trials of the apartment protocol with a nondisabled subject wearing the PAM. During each trial, the device failed to register distance measurements despite the subject's adequate ambulation speed.

The reduced accuracy of the DW in the apartment setting may be related to its internal switch mechanism. The DW uses a cantilever arm switch that requires acceleration of $\geq 0.35 g$ to register a step [17]. Any small or less forceful step not resulting in this level of acceleration would not be counted by the device. The variable nature of the activities performed in the apartment setting likely resulted in certain steps' not meeting the acceleration threshold of the switch mechanism and thus not being recorded by the device.

In the gymnasium, both the DW and the PAM were significantly more accurate in determining step count. All subjects were observed to walk with more continuity and less step-to-step variability during the gymnasium protocol. In nondisabled subjects, the Yamax SW series had demonstrated very good accuracy, up to 97.0 percent for 
step count and 93.8 percent for distance during track walking [12]. Our findings indicate that the DW was capable of measuring step count and distance at almost equally accurate levels in K3 transtibial amputation subjects under comparable testing circumstances. The incorporation of a turn after each length of the gymnasium in our protocol was included to simulate some of the directional changes that often occur during daily walking. The incorporation of simple changes in direction did not introduce clinically relevant step count errors into the measurements of the DW.

Step count accuracy has been more accurately demonstrated by the step activity monitor (SAM) (Prosthetics Research Study; Seattle, Washington), a two-dimensional accelerometer that has been validated across a wide range of gait styles, including that of people who have undergone lower-limb amputation. The SAM has been shown to detect steps with better than 99 percent accuracy [3]. However, the slightly increased accuracy with the SAM does not seem to outweigh its disadvantages of needing minor customization for each individual and high cost (U.S. \$2,100-price includes docking station and one activity monitor).

Bussmann et al. reported that the PAM was 95.8 percent accurate for distance ambulated continuously overground at optimal walking speeds and 98.4 percent accurate for step count [16]. Although the accuracy of step count and ambulation measurements in our study were slightly lower than these values, we consider the PAM's accuracy during relatively continuous ambulation to be acceptable. The minor reductions in accuracy may be a result of incorporating direction changes into our testing protocol, compared with the continuous ambulation in the protocol used by Bussmann et al.

Reports on the effects of obesity on pedometer accuracy have varied. Shepherd et al. reported reduced step count accuracy in obese individuals (BMI $>30 \mathrm{~kg} / \mathrm{m}^{2}$ ) [2]. Schneider et al. [12] and Swartz et al. [18] found that neither BMI nor device placement (front, side, or back) significantly affected the accuracy of a Yamax SW series pedometer. In our study, we noted that abdominal obesity was often a cause of less than optimal vertical position of the DW over the anterior superior iliac spine. Placement of the DW to a more lateral position at the waistline often compensated for this and improved device accuracy. Although the step count of the DW was statistically less accurate for obese subjects, the device maintained adequate monitoring accuracy, at approximately 90 percent in obese subjects during relatively continuous ambulation.
The ambulation monitoring capabilities of the DW and PAM are superior during more consistent and continuous ambulation (as was seen in our gymnasium protocol) compared with the varied forms of movement that occur within a small indoor environment (as in our apartment protocol). In an environment without significant barriers such as steps and curbs, both devices proved to be appropriate for monitoring activity of transtibial amputation subjects ambulating at the K3 level. The addition of environmental barriers and varied terrain may introduce additional measurement errors; therefore, generalization to actual outdoor community ambulation monitoring cannot be made based on our study results.

Considerable differences in gait characteristics may exist in lower K-level transtibial amputees and those with anatomically higher limb amputations. Our population of K3 level transtibial amputees often represents a physically active group with varied activities, gait patterns, speeds, and limitations that are quite different than those at lower K-levels. We feel this limits generalization of our finding to other K-levels, especially $\mathrm{K} 1$ and $\mathrm{K} 2$, where people will not likely be able to walk fast enough for the devices to be accurate. As well, only two participants used a gait aid during the gymnasium protocol and no participant used a gait aid in the apartment. Therefore, the effect of a gait aid could not be assessed in our study. Lower K-level amputees will be more likely to rely on a gait aid and walk in a manner that may introduce additional inaccuracies in the devices tested.

Either device presents its own unique advantages for use in the amputee population. The DW is simple to use and inexpensive, at approximately U.S. \$40 compared with U.S. $\$ 1,100$ for the PAM. The average daily step count in those having undergone amputation has not been definitively established in the literature. A few studies looking at factors that may influence ambulation activity in lower-limb amputees have found daily step counts ranging from 1,000 to 5,000 [19-20]. People with other disabilities and chronic diseases have been reported to take 3,500 to 5,500 steps/day [21]. The DW can measure up to 99,999 steps per monitoring session and could therefore potentially be used for a longer-duration study than the PAM, which is currently limited to 1 week of data collection. The PAM offers the advantages of additional activity measurement parameters above step count and ambulation distance. Also, since it remains strapped to the prosthesis, the likelihood of compliance in a study or clinical setting is higher, whereas the DW may unintentionally not be worn from one day to the next. Lastly, 
the PAM may be a better option for use in obese people, since in our study the accuracy of step count and ambulation distance with this device was not aversely affected in this subgroup.

Our study design presented some analytical limitations. The lack of associations between the accuracy of the devices in certain settings and for certain subject characteristics may be related to large variability but relatively small differences between mean device accuracy. The large variability in step count and distance measurements is likely more related to individual differences in stride length as opposed to variability in how well the device worked for a particular individual. These differences are more obvious when studying a small group. Other studies have found similar amounts of variability in step count when assessing a relatively small population such as ours $(n<30)$ [22-23]. A sample size of 177 subjects would provide adequate statistical power $(\beta=$ 0.8 ) for all analyses.

\section{CONCLUSIONS}

Accurate and objective devices that quantify activity levels in subjects with lower-limb amputation can benefit patient care research. In our study, the DW and PAM were equally accurate and appropriate for monitoring step count during relatively continuous ambulation in $\mathrm{K} 3$ unilateral transtibial amputation subjects in settings without environmental barriers. The DW was significantly more accurate than the PAM for distance measurement. Both devices demonstrated serious limitations for ambulation monitoring during tasks performed in an indoor household setting. We conclude that these devices are appropriate only for measuring relatively continuous ambulation. Further study is needed to clarify the use of the DW and the PAM in other populations and other settings. These studies should include (1) assessments in a person's own environment with improved contextual task identification, (2) assessments of different K-level ambulators, and (3) assessments of outdoor ambulation tasks.

\section{ACKNOWLEDGMENTS}

We acknowledge Tomy Thomas and Pamela Miller for their contributions toward data collection and analysis and Gloria Baker for editing the manuscript.
We are thankful to Össur for providing the PAM for the study. Össur did not have any involvement in the study design; data collection, analysis, or interpretation; and writing or submission of this article.

This material is the result of work supported with resources and the use of facilities at the Institute for Rehabilitation Research and Development at The Rehabilitation Centre and the University of Ottawa Summer Studentship Medical Program.

The authors have declared that no competing interests exist.

\section{REFERENCES}

1. Holden J, Fernie GR, Soto M. An assessment of a system to monitor the activity of patients in a rehabilitation programme. Prosthet Orthot Int. 1979;3(2):99-102.

[PMID: 503802]

2. Shepherd EF, Toloza E, McClung CD, Schmalzried TP. Step activity monitor: Increased accuracy in quantifying ambulatory activity. J Orthop Res. 1999;17(5):703-8. [PMID: 10569479]

3. Coleman KL, Smith DG, Boone DA, Joseph AW, Del Aguila MA. Step activity monitor: Long-term, continuous recording of ambulatory function. J Rehabil Res Dev. 1999; 36(1):8-18. [PMID: 10659890]

4. Bussmann JB, Grootscholten EA, Stam HJ. Daily physical activity and heart rate response in people with a unilateral transtibial amputation for vascular disease. Arch Phys Med Rehabil. 2004;85(2):240-44. [PMID: 14966708]

5. Centers for Medicare and Medicaid Services. U.S. Department of Health and Human Services. HCFA Common Procedure Coding System (HCPCS) 2001. Springfield (VA): U.S. Department of Commerce, National Technical Information Service; 2001. Chapter 5.3.

6. Tarlov AR, Ware JE Jr, Greenfield S, Nelson EC, Perrin E, Zubkoff M. The Medical Outcomes Study. An application of methods for monitoring the results of medical care. JAMA. 1989;262(7):925-30. [PMID: 2754793]

7. Day HJ. The assessment and description of amputee activity. Prosthet Orthot Int. 1981;5(1):23-28. [PMID: 7279610$]$

8. Franchignoni F, Orlandini D, Ferriero G, Moscato TA. Reliability, validity, and responsiveness of the locomotor capabilities index in adults with lower-limb amputation undergoing prosthetic training. Arch Phys Med Rehabil. 2004;85(5): 743-48. [PMID: 15129398]

9. Brandes M, Rosenbaum D. Correlations between the step activity monitor and the DynaPort ADL-monitor. Clin Biomech (Bristol, Avon). 2004;19(1):91-94.

[PMID: 14659936] 
10. Miller WC, Deathe AB, Speechley M. Lower extremity prosthetic mobility: A comparison of 3 self-report scales. Arch Phys Med Rehabil. 2001;82(10):1432-40. [PMID: 11588750]

11. Wilde BE, Sidman CL, Corbin CB. A 10,000-step count as physical activity target for sedentary women. Res Q Exerc Sport. 2001;72(4):411-14. [PMID: 11770790]

12. Schneider PL, Crouter SE, Lukajic O, Bassett DR Jr. Accuracy and reliability of 10 pedometers for measuring steps over a 400-m walk. Med Sci Sports Exerc. 2003;35(10): 1779-84. [PMID: 14523320]

13. Schneider PL, Crouter SE, Bassett DR. Pedometer measures of free-living physical activity: Comparison of 13 models. Med Sci Sports Exerc. 2004;36(2):331-35. [PMID: 14767259]

14. Kochersberger G, McConnell E, Kuchibhatla MN, Pieper C. The reliability, validity, and stability of a measure of physical activity in the elderly. Arch Phys Med Rehabil. 1996;77(8):793-95. [PMID: 8702373]

15. Bussmann HB, Reuvekamp PJ, Veltink PH, Martens WL, Stam HJ. Validity and reliability of measurements obtained with an "activity monitor" in people with and without transtibial amputation. Phys Ther. 1998;78(9):989-98. [PMID: 9736896]

16. Bussmann JB, Culhane KM, Horemans HL, Lyons GM, Stam HJ. Validity of the prosthetic activity monitor to assess the duration and spatio-temporal characteristics of prosthetic walking. IEEE Trans Neural Syst Rehabil Eng. 2004;12(4):379-86. [PMID: 15614993]
17. Tudor-Locke C, Ainsworth BE, Thompson RW, Matthews CE. Comparison of pedometer and accelerometer measures of free-living physical activity. Med Sci Sports Exerc. 2002;34(12):2045-51. [PMID: 12471314]

18. Swartz AM, Bassett DR Jr, Moore JB, Thompson DL, Strath SJ. Effects of body mass index on the accuracy of an electronic pedometer. Int J Sports Med. 2003;24(8):588-92. [PMID: 14598195]

19. Holden JM, Fernie GR. Extent of artificial limb use following rehabilitation. J Orthop Res. 1987;5(4):562-68. [PMID: 3681530]

20. Klute GK, Berge JS, Orendurff MS, Williams RM, Czerniecki JM. Prosthetic intervention effects on activity of lower-extremity amputees. Arch Phys Med Rehabil. 2006; 87(5):717-22. [PMID: 16635636]

21. Tudor-Locke CE, Myers AM. Methodological considerations for researchers and practitioners using pedometers to measure physical (ambulatory) activity. Res Q Exerc Sport. 2001;72(1):1-12. [PMID: 11253314$]$

22. Welk GJ, Differding JA, Thompson RW, Blair SN, Dziura J, Hart P. The utility of the Digi-Walker step counter to assess daily physical activity patterns. Med Sci Sports Exerc. 2000;32(9 Suppl):S481-88. [PMID: 10993418]

23. Hartsell H, Fitzpatrick D, Brand R, Frantz R, Saltzman C. Accuracy of a custom-designed activity monitor: Implications for diabetic foot ulcer healing. J Rehabil Res Dev. 2002; 39(3):395-400. [PMID: 12173759]

Submitted for publication May 16, 2007. Accepted in revised form August 9, 2007. 